УДК 52-5

A.B. Торопов, канд. техн. наук, доц. ORCID 0000-0001-8505-0266

Л.В.Торопова, асс. ORCID 0000-0001-6882-2295

Національний технічний університет України «Київський політехнічний інститут імені Ігоря Сікорського»

\title{
КВАЗИОПТИМАЛЬНОЕ УПРАВЛЕНИЕ ЗАГРУЗКОЙ КОНВЕЙЕРА НА ОСНОВЕ МИНИМАКСНОГО КРИТЕРИЯ КАЧЕСТВА
}

В работе была решена задача синтеза квазиоптимального регулятора, обеспечивающего минимизацию отклонения загрузки конвейера при минимуме энергетических затрат на управление. При осуществлении процедуры синтеза использовалась математическая модель системы стабилизации загрузки конвейера с учетом чистого запаздывания по перемещению груза и колебательном характере переходного процесса в контуре скорости. Для нахождения квазиоптимального управления использовался метод Айзекса - Беллмана, позволяющего найти минимум функционала качества при наихудшем влиянии возмущающих воздействий. Задача решалась при условии существованя «седловой» точки минимаксного критерия оптимальности по управляющему и возмущающим воздействиям. В результате синтеза был получен закон квазиоптимального управления в виде суммы линейных составляющих от переменных состояния. Исследование методом ичифрового моделирования показало преимущества полученного алгоритма управления по сравнению $с$ классическими регуляторами. Полученный закон квазиоптимального управления достаточно просто может быть реализован на программируемом контроллере для управления конвейером.

Ключевые слова: загрузка конвейера, транспортировка сыпучих материалов, метод Айзекса Беллмана, минимаксный функционал качества, седловая точка.

Введение. В настоящее время на предприятиях актуальной является задача энергосбережения за счет оптимизации работы систем с регулируемым электроприводом. Одними из наиболее энергоемких электроприводов со значительным потенциалом энергосбережения являются конвейерные системы непрерывной транспортировки сыпучих грузов, таких как песок, уголь, зерно. Применение регулируемых электроприводов позволяет стабилизировать загрузку конвейера, обеспечить максимальную производительность, увеличить надежность и долговечность системы в целом. Важно отметить, что именно стабилизация количества груза на конвейере позволяет получить максимальную энергоэффективность оборудования.

В то же время существенной проблемой внедрения таких систем является сложность их наладки и эксплуатации. Дело в том, что наличие чистого запаздывания в контуре регулирования делает неэффективным применение стандартных регуляторов, получаемых методами последовательной коррекции, либо частотными методами. Отклонения реальной загрузки конвейера от заданной приводят к перегрузке приводных двигателей, их перегреву, а также излишнему потреблению системой управления электроэнергии.

Цель и задачи исследования. Получение наилучшего режима работы конвейера с точки зрения энергоэффективности возможно за счет применения регуляторов, полученных путем применения различных методов оптимизации. Одним из наиболее эффективных методов синтеза оптимальных регуляторов является метод Беллмана - Ляпунова. В этом случае закон управления для системы с чистым запаздыванием и случайным возмущающим воздействием будет содержать коэффициенты, зависящие от времени. Процедура поиска аналитического выражения для этих коэффициентов с вычислительной точки зрения является крайне трудоемкой и приведет к значительному усложнению системы управления. Поэтому, разработка системы управления загрузкой конвейера, обеспечивающая оптимальное поведение системы и относительно простой с точки зрения практической реализации, является актуальной задачей.

Материал и результаты исследования. Задача регулирования загрузки конвейеров для снижения энергопотребления предприятий с системами транспортировки сыпучих материалов широко

(C) А.В. Торопов, Л.В.Торопова, 2018 


\section{ISSN 1813-5420 (Print). Енергетика: економіка, технології, екологія. 2018. № 1}

рассматривается в работах [1-5]. В них решается задача синтеза линейно - квадратичных регуляторов загрузки, в которой существующие нелинейности рассматриваются как минимизируемые управляющие воздействия. Также в качестве корректирующих воздействий рассматриваются и внешние возмущения, действующие на контур. Особенностью существующих решений является то, что в качестве минимизируемого функционала качества используется классический квадратичный критерий оптимальности. В этом случае ограничения на возмущающие воздействия подразумевают их минимизацию, то есть фактически, задача поиска оптимального регулятора будет доставлять экстремум функционалу качества лишь при минимальном влиянии возмущений и нелинейностей [6]. Следовательно, полученные ранее, решения задачи аналитического конструирования регуляторов дают наилучший результат лишь при незначительных колебаниях загрузки, и не обеспечивают низкую чувствительность к вариациям возмущений.

Альтернативным подходом к обеспечению оптимального поведения системы является использование инвариантного управления, нечувствительного к внешним возмущающим воздействиям. Существует большое количество вариаций процедуры Беллмана - Ляпунова для решения задачи нахождения робастных регуляторов, из которых одним из наиболее популярных является метод Айзекса - Беллмана [7].

При использовании метода, предложенного в [8], задача поиска оптимального регулятора сводится к дифференциальной игре с двумя участниками, где первый игрок в виде управляющего воздействия будет обеспечивать минимум функционала качества при наихудшем характере возмущающих воздействий, формируемым вторым игроком. Такой подход позволит обеспечить высокую робастность системы к колебаниям количества сыпучего груза, которое поступает на конвейер.

Осуществим процедуру синтеза оптимального регулятора загрузки конвейера, электромеханическая система которого описывается колебательным звеном. Такое допущение будет справедливым для однодвигательного конвейера с настройкой контура скорости привода на технический оптимум. Предложенный подход позволит существенно упростить процедуру синтеза без значительного снижения точности решения задачи оптимизации.

Контур стабилизации загрузки непрерывного транспорта для сыпучих грузов с учетом предложенной модели электромеханической системы примет вид, представленный на рис.1.

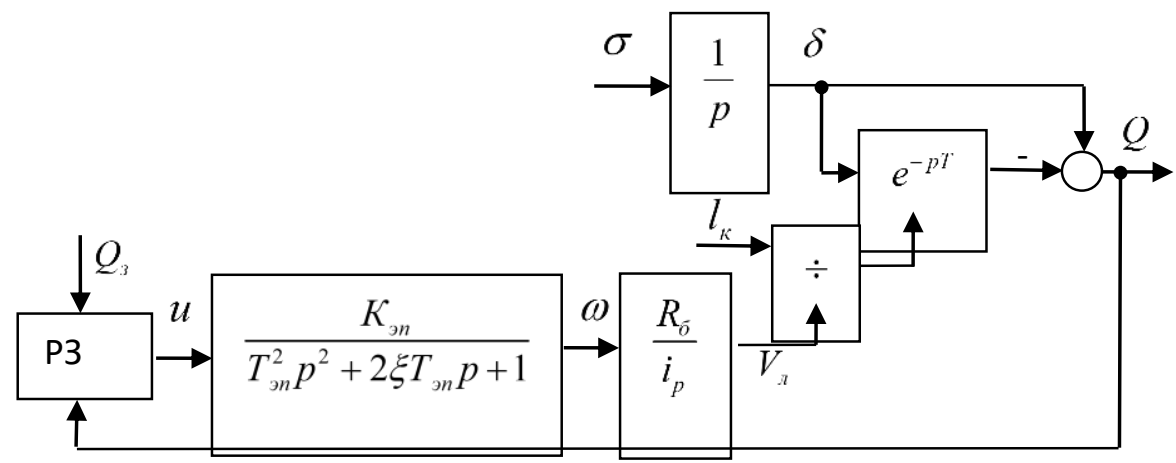

Рисунок 1 - Схема контура стабилизации загрузки конвейера горнодобывающего предприятия.

На рис.1 введены следующие обозначения: $Q_{3}, Q$ - заданное и реальное количество груза на конвейере в текущий момент времени; $\delta$ - количество груза, загружаемого на конвейер; $\sigma$ - мгновенное количество груза, поступающее на конвейер; $l_{\kappa}$ - длина конвейера; $R_{\sigma}$ - радиус приводного барабана; $i_{p}$ - передаточное число редуктора; $K_{э n}, \xi, T_{\text {эn }}$ - параметры электромеханической системы конвейера при настройке внешнего контура на модульный оптимум; $V$ - линейная скорость перемещения ленты; $\omega$ скорость вращения двигателя приводного барабана; $u$ - управляющее воздействие.

Зависимость текущей загрузки конвейера от количества груза, поступающего на ленту, определяется выражением (1):

$$
Q=\left(1-e^{-p T}\right) \delta
$$

Разложим составляющую $e^{p T}$ в ряд Тейлора, ограничимся двумя составляющими, и при учете интегральной составляющей нагрузки получим: 


$$
Q=\left(1-e^{-p T}\right) \delta=\left(1-\frac{1}{e^{p T}}\right) \delta=\left(\frac{e^{p T}-1}{e^{p T}}\right) \delta \approx\left(\frac{1+p T-1}{1+p T}\right) \delta=\frac{T}{T p+1} \sigma .
$$

Поскольку время задержки зависит от скорости в зависимости $T=l_{\kappa} i_{p} R_{\sigma}^{-1} \omega^{-1}$, можем записать в нормальной форме Коши уравнение, описывающее изменение загрузки:

$$
\frac{d Q}{d t}=-\frac{R_{\sigma}}{l_{\kappa} i_{p}} \omega Q+\sigma
$$

Поведение привода, при условии его настройки внешнего контура регулирования скорости на технический оптимум, описывается системой дифференциальных уравнений:

$$
\begin{aligned}
& \frac{d \omega}{d t}=\varepsilon \\
& \frac{d \varepsilon}{d t}=-\frac{1}{T_{\ni}^{2}} \omega-\frac{2 \xi}{T_{\ni n}}+\frac{K_{\ni n}}{T_{\ni n}} u .
\end{aligned}
$$

Получим в итого систему дифференциальных уравнений в нормальной форме Коши, описывающее поведение конвейерной системы:

$$
\begin{aligned}
& \dot{x}_{1}=-a_{1} x_{1} x_{2}+\sigma, \\
& \dot{x}_{2}=x_{3}, \\
& \dot{x}_{3}=-a_{2} x_{2}-a_{3} x_{3}+b u,
\end{aligned}
$$

где $x_{1}=Q, x_{2}=\omega, a_{1}=l_{k}^{-1} R_{\delta} i_{p}^{-1}, a_{2}=T_{э n}^{-1}, b=K_{э n} T_{э n}^{-1}$.

Минимаксный функционал качества сформируем, исходя из требования динамической точности и минимума энергетических затрат на управление, а также максимизации возмущающего воздействия [9]:

$$
\min _{u} \max _{\sigma} J=\int_{0}^{\infty}\left(q_{1} x_{1}^{2}+q_{2} x_{2}^{2}+q_{3} x_{3}^{2}+r_{1} u^{2}-r_{2} \sigma^{2}\right) d t
$$

Наличие в функционале качества составляющей $-r_{2} \sigma^{2}$ указывает, что возмущающие воздействие меняется по линейной зависимости от переменных состояния, однако наихудшим образом по отношению к управляющему воздействию в произвольный момент времени.

Процедура решения прикладных задач аналитического конструирования квазиоптимального регулятора на основе теории дифференциальных игр [7,8] предполагает наличие «седловой» точки минимизируемого функционала, то есть существование решение игровой задачи. Поэтому, полагаем, что существуют допустимые $u$ и $\sigma$, которые доставляют седловую точку выбранному функционалу качества.

Далее осуществим линеаризацию составляющей $x_{1} x_{2}$, входящей в первое дифференциальное уравнение системы. При этом решение задачи оптимизации станет приближенным, то есть решение, полученное в результате синтеза, будет квазиоптимальным.

Существует два основных подхода к проблеме линеаризации. В первом случае медленно меняющаяся составляющая принимается детерминированной, то есть $x_{1} x_{2}=K\left(x_{1}\right) x_{2}$ [10]. Здесь $K\left(x_{1}\right)$ - коэффициент, принимаемый постоянным для некоторой области фазового пространства. Во втором случае осуществляется линеаризация «в точке» [11], например, в точке номинального режима работы конвейера. При этом $x_{1} x_{2}=a_{1}\left(x_{1}, x_{2}\right) x_{1}+a_{2}\left(x_{1}, x_{2}\right) x_{2}$, где $a_{1}\left(x_{1}, x_{2}\right), a_{2}\left(x_{1}, x_{2}\right)$ - коэффициенты линеаризации «в точке». Для задачи максимизации загрузки конвейера второй подход является более предпочтительным. В этом случае линеаризованная система дифференциальных уравнений, описывающая динамику конвейера, запишется: 


$$
\begin{aligned}
& \dot{x}_{1}=-a_{11} x_{1}-a_{12} x_{2}+\sigma, \\
& \dot{x}_{2}=x_{3}, \\
& \dot{x}_{3}=-a_{2} x_{2}-a_{3} x_{3}+b u,
\end{aligned}
$$

где $a_{11}, a_{12}$ - коэффициенты линеаризации «в точке».

Для линеаризованной системы дифференциальных уравнений и выбранного минимаксного функционала качества уравнение Айзекса - Беллмана запишется:

$$
\begin{aligned}
& \min _{u} \max _{\sigma}\left[q_{1} x_{1}^{2}+q_{2} x_{2}^{2}+q_{3} x_{3}^{2}++r_{1} u^{2}-r_{2} \sigma^{2}+\right. \\
& \left.+\frac{\partial V}{\partial x_{1}}\left(-a_{11} x_{1}-a_{12} x_{2}+\sigma\right)+\frac{\partial V}{\partial x_{2}} x_{3}+\frac{\partial V}{\partial x_{3}}\left(-a_{2} x_{2}-a_{3} x_{3}+b u\right)\right]=0 .
\end{aligned}
$$
получим:

Произведем поиск экстремума функционала по управляющему и возмущающему воздействию,

$$
u=-\frac{b}{2 r_{1}} \frac{\partial V}{\partial x_{3}} ; \sigma=\frac{1}{2 r_{2}} \frac{\partial V}{\partial x_{1}}
$$

Осуществим замыкание системы путем подстановки полученных выражений в функциональное уравнение (8), получим уравнение Айзекса - Беллмана в замкнутой форме:

$$
\begin{aligned}
& q_{1} x_{1}^{2}+q_{2} x_{2}^{2}+q_{3} x_{3}^{2}+\frac{\partial V}{\partial x_{1}}\left(-a_{11} x_{1}-a_{12} x_{2}\right)+\frac{\partial V}{\partial x_{2}} x_{3}+ \\
& +\frac{\partial V}{\partial x_{3}}\left(-a_{2} x_{2}-a_{3} x_{3}\right)=\frac{b^{2}}{4 r_{1}}\left(\frac{\partial V}{\partial x_{3}}\right)^{2}-\frac{1}{4 r_{2}}\left(\frac{\partial V}{\partial x_{1}}\right)^{2}
\end{aligned}
$$

Решением данного уравнения будет функция $V$ в виде квадратичной формы от переменных состояния системы вида $[9,10]$ :

$$
V=K_{11} x_{1}^{2}+2 K_{12} x_{1} x_{2}+2 K_{13} x_{1} x_{3}+K_{22} x_{2}^{2}++2 K_{23} x_{2} x_{3}+K_{33} x_{3}^{2}
$$

Подставим выражения для частных производных в уравнения Айзекса - Беллмана в замкнутой форме и выделив коэффициенты при одинаковых степенях, получим систему алгебраических уравнений Риккати [9]. Из нее получим значения коэффициентов функции Беллмана и подставим их в исходные выражения для управления и возмущения:

$$
u=-K_{1 u} x_{1}-K_{2 u} x_{2}-K_{3 u} x_{3} ; \sigma=K_{1 \sigma} x_{1}+K_{2 \sigma} x_{2}+K_{3 \sigma} x_{3}
$$

Исследование динамических характеристик контура регулирования загрузки было проведено методом цифрового моделирования с применением пакета MATLAB и его приложения Simulink. B качестве объекта рассматривался конвейер горнодобывающего предприятия с рекомендуемой загрузкой 45 тонн и длиной 500 метров. Переменные состояния $x_{2}, x_{3}$ были получены в виде разности задания, получаемого от эталонной модели привода конвейера, настроенного на модульный оптимум. Задание по загрузке формировалось в виде обратно - пропорционального сигнала по скорости конвейера с целью максимальной компенсации чистого запаздывания ленты.

Графики переходных процессов по загрузке конвейера при использовании линейно - квадратичного регулятора, синтезируемого при учете наилучшего характера возмущающих воздействий, представлены на рисунке 2. В режиме стабилизации выходной величины имеются существенные колебания загрузи до $10 \%$ от заданного значения. 


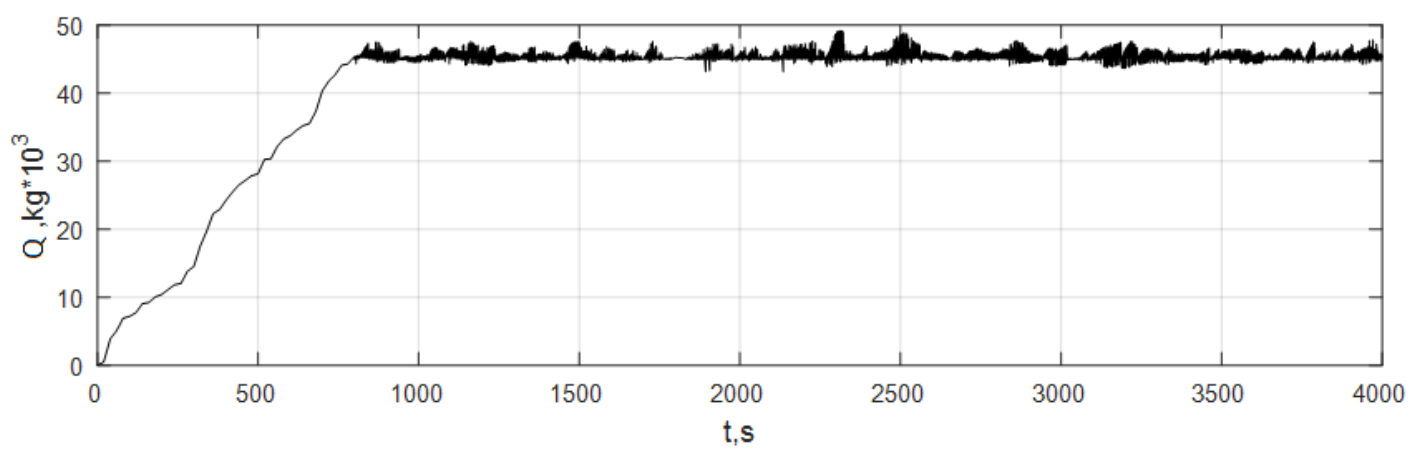

Рисунок 2 - График переходного процесса по загрузке при использовании линейно - квадратичного регулятора.

График переходных процессов по загрузке конвейера при использовании синтезированного квазиоптимального регулятора представлены на рисунке 3. При использовании синтезированного регулятора скачок загрузки при заполнении конвейера практически отсутствует, колебания загрузки в режиме стабилизации также существенно уменьшились.

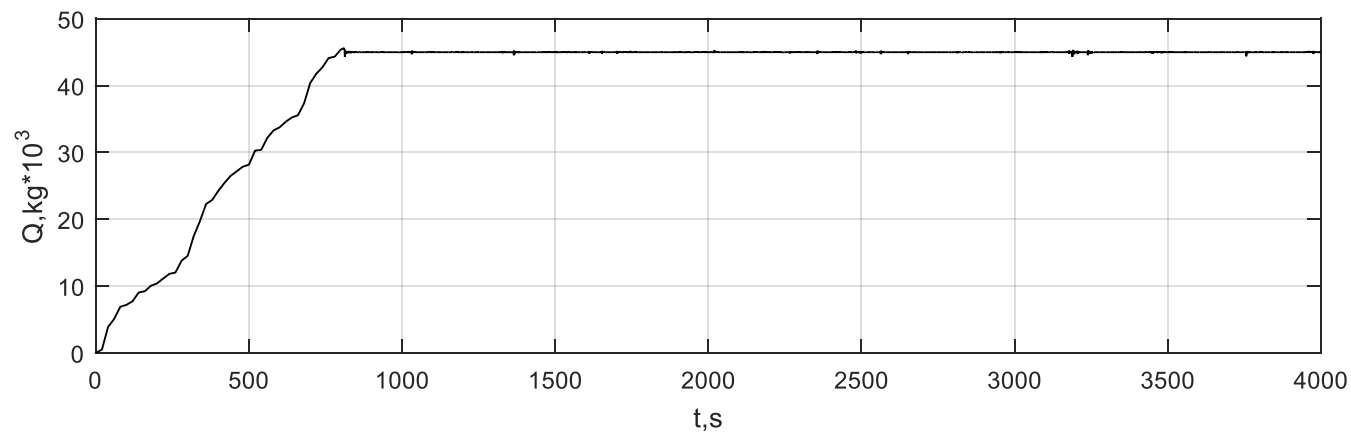

Рисунок 3 - График переходного процесса по загрузке при использовании квазиоптимального регулятора.

Синтезированный квазиоптимальный регулятор, коэффициенты которого были определены с учетом наихудшего характера возмущающих воздействий, в отличие от линейно - квадратичного регулятора, лучше компенсирует случайные колебания загрузки в режиме стабилизации. При этом сложность регулятора существенно не увеличилась и полученный алгоритм управления, а также блок формирования задания по переменным состояния может быть реализован на микропроцессорном устройстве регулирования.

Выводы. Использование метода Айзекса - Беллмана при игровой постановке задачи синтеза позволило разработать алгоритм управления, обеспечивающий оптимальное качество управления при наихудшем характере возмущающих воздействий. Линеаризация «в точке», а также использование детерминированных коэффициентов регулятора обеспечили квазиоптимальное управление загрузкой без значительного усложнения системы регулирования. Сравнение результатов моделирования показало преимущество предложенного синтезированного регулятора, по сравнению с линейно - квадратичными, применяемыми в существующих конвейерных системах. Полученный алгоритм управления, а также используемый блок формирования заданий по переменным состояния может быть реализован на общепромышленном программируемом логическом контроллере, используемом для решения задач автоматизации. Предложенный подход к формированию контура регулирования загрузки предусматривает использование датчиков объема сыпучего груза, подключаемых к контроллеру по одному из промышленных интерфейсов. Дальнейшее улучшение качества регулирования загрузки возможно путем более подробного учета динамических свойств конвейерной системы, что может привести, в свою очередь, к существенному усложнению алгоритма управления.

\section{Список использованной литературы}

1. Дмитриева В.В. Разработка и исследование системы автоматической стабилизации погонной нагрузки магистрального конвейера/Дис.....кандидата техн.наук 05.13.06./В.В. Дмитриева; Ва Московский государственный горный университет- Москва,2005. - 162c. 


\section{ISSN 1813-5420 (Print). Енергетика: економіка, технології, екологія. 2018. № 1}

2. Боровикова А.П. Разработка и исследование системы автоматического управления загрузкой магистральной конвейерной линии/ А.П. Боровикова, А.Е. Ткаченко, К.Н. Маренич// Автоматизация технологических объектов и процессов. Поиск молодых: сборник научных трудов XVII научнотехнической конференции аспирантов и студентов в г. Донецке 24-25 мая 2017 г. - Донецк : ДонНТУ, 2017. $-409 \mathrm{c}$.

3. Дмитриева, В. В. Автоматическая стабилизация погонной нагрузки ленточного конвейера / В. В. Дмитриева, Л. Д. Певзнер // Московский государственный горный институт. - Москва : Препринт, 2004. $25 \mathrm{c}$.

4. Дмитриева В.В. Поддержание величины тягового фактора ленточного конвейера с двухдвигательным приводом /В.В. Дмитриева, Куанг Пьей// 2015/ Горный информационно-аналитический бюллетень (научно-технический журнал), 2015-№6 с.189-198.

5. Печеник М. В. Дослідження втрат енергії в електромеханічній системі конвеєра при плавній зміні навантаження / М. В. Печеник, С. О. Бур'ян, Л. М. Наумчук // Електромеханічні і енергозберігаючі системи. - 2015. - Вип. 2. - С. 67-73

6. Летов А.М. Динамика полета и управление. - М.: Наука, 1969. - 359 с.

7. Атанс М.,Фалб П.Л. Оптимальное управление.М.: Машиностроение, 1968, - 764c.

8. Жуковский В.И., Чикрий А.А. Линейно-квадратичные дифференциальные игры. К.: Наукова думка, 1994.- 314c.

9. Kudin V.F. Synthesis of a Robust Suboptimal Controller Based on the Optimality Minimax Criteria/ V.F. Kudin, Ye. Ye. Alexandrov//IFAC Proceedings Volumes. Vol. 25, Issue 21, September 1992- p.282-283.

10. Kudin, V. F. Suboptimal control of crane moving mechanism considering damping of load / V. F. Kudin, M. V. Pechenik, S. P. Kolesnichenko // 16 International Conference on Soft Computing MENDEL 2010, Brno, Czech Republic, June 23-25. - P. 381-386.

11. Петраков Ю.В. Автоматичне управління процесами обробки матеріалів різанням Київ: УкрНДІАТ, 2003.- 383 с.

A. Toropov, Cand. Sc. (Eng.), assoc. prof. ORCID 0000-0001-8505-0266

L. Toropova, TF ORCID 0000-0001-6882-2295

National Technical University of Ukraine «Igor Sikorsky Kyiv Polytechnic Institute»

\section{QUASI-OPTIMAL CONTROL OF CONVEYOR LOADING BASED ON MINIMAX QUALITY CRITERIA}

In the work, the problem of synthesis of quasi-optimal controller, which minimizes the deviation of the conveyor load with a minimum of energy losses, was solved. When performing the synthesis procedure, a mathematical model of the conveyor loading stabilization system, taking into account the delay link in the load movement and the oscillating behavior of the transient process in the speed loop, was used. To find quasi-optimal control, the Isaacs-Bellman method, which makes it possible to find a minimum of the quality functional with the worst behavior of disturbing influences, was used. The problem was solved under the condition that there existed a «saddle» point of the minimax optimality criterion for control and disturbing influences. As a result of the synthesis, the law of quasi-optimal control was obtained as the sum of the linear components of the state variables. The investigation using method of digital simulation shows the advantages of the obtained control algorithm in comparison with the classical regulators. The obtained law of quasi-optimal control can be easily implemented based on a programmable regulator for conveyor control.

Keywords: conveyor loading, conveying of bulk materials, Isaacs - Bellman method, minimax functional of quality, «saddle» point.

\section{References}

1.Dmitrieva V.V. Development and research of the system of automatic stabilization of the running load of the main conveyor / Dis .... candidate of technical sciences 05.13.06./V.V. Dmitrieva; Moscow State Mining University- Moscow, 2005. - 162c. (Rus.) 
2.Borovikova A.P., Tkachenko A.E., Marenich K.N. Development and investigation of the automatic control system for loading the main conveyor line// Automation of technological objects and processes. Collection of scientific papers of the XVII scientific and technical conference of graduate students and students in Donetsk May 24-25, 2017 - Donetsk: DonNTU, 2017. - 409 p.

3.Dmitrieva V. V., Pevzner L. D. Automatic stabilization of the load of a belt conveyor // Moscow State Mining Institute. - Moscow: Preprint, 2004. - 25 p. (Rus.)

4.Dmitrieva V.V., Quang Pey Maintaining the value of traction factor of a belt conveyor with a two-motor drive //Mining informational and analytical bulletin (scientific and technical journal), 2015, №6 p.189-198. (Rus.)

5.Pechenik M.V., Buryan S.O., Naumchuk L. M. Investigation of energy losses in the electromechanical system of the conveyor under a smooth load change // Electromechanical and energy saving systems. - 2015. Vol. 2. - p. 67-73 (Ukr.)

6.Letov A.M. Flight dynamics and control. - Moscow: Nauka, 1969. - 359 p. (Rus.)

7.Atans M., Falb P.L. Optimalnoe upravlenie.M .: Mashinostroenie, 1968, - 764p. (Rus.)

8.Zhukovsky V.I., Chikriy A.A. Linear-quadratic differential games. K .: Naukova dumka, 1994.- 314p. (Rus.)

9.Kudin V.F., Alexandrov Ye. Ye. Synthesis of a Robust Suboptimal Controller Based on the Optimality Minimax Criteria//IFAC Proceedings Volumes. Vol. 25, Issue 21, September 1992- p.282-283. (Eng.).

10. Kudin, V. F., Pechenik M. V., Kolesnichenko S. P. Suboptimal control of crane moving mechanism considering damping of load // 16 International Conference on Soft Computing MENDEL 2010, Brno, Czech Republic, June 23-25. - P. 381-386. (Eng.)

11. Petrakov Yu.V. Automatic control of the processes of cutting materials processing// Kyiv: UkrNIAT, 2003.- 383 p. (Ukr.)

A.B. Торопов, канд. техн. наук, доц. ORCID 0000-0001-8505-0266

Л.В.Торопова, асист. ORCID 0000-0001-6882-2295

Національний технічний університет України «Київський політехнічний інститут імені Ігоря Сікорського»

\section{КВАЗИОПТИМАЛЬНЕ КЕРУВАННЯ ЗАВАНТАЖЕННЯМ КОНВЕСРА НА ОСНОВІ МІНІМАКСНОГО КРИТЕРІЮ ЯКОСТІ}

В роботі вирішена задача синтезу квазіоптимального регулятора, щзо забезпечує мінімізацію відхилення завантаження конвеєра, при мінімумі енергетичних затрат на управління. При здійсненні прочедури синтезу використовувалася математична модель системи стабілізації завантаження конвеєра, з урахуванням чистого запізнювання по перемішенню вантажу $i$ коливальному характері перехідного процессу у контурі швидкості. Для знаходження квазіоптимального управління використовувався метод Айзекса - Беллмана, щчо дозволяє знайти мінімум функиіоналу якості при найгіршому характері збурюючих впливів. Задача вирішувалась за умови існування «сідлової» точки минимаксного критерію оптимальності за керуючим та збурюючими впливами. В результаті синтезу було отримано закон квазіоптимального управління у вигляді суми лінійних складових від змінних стану. Дослідження методом ичифрового моделювання показало переваги отриманого алгоритму управління у порівнянні з класичними регуляторами. Отриманий закон квазіоптимального управління досить просто може бути реалізований на програмованому контролері управління конвеєром.

Ключові слова: завантаження конвеєра, транспортування сипучих матеріалів, метод Айзекса Беллмана, мінімаксний функціонал якості, сідлова точка. 